\title{
Modified breast cancer model for preclinical immunotherapy studies
}

Eriko Katsuta ${ }^{1,2,3}$, Stephanie C. DeMasi ${ }^{1,2}$, Krista P. Terracina ${ }^{1,2}$, Sarah Spiegel ${ }^{2}$, Giao Q. Phan ${ }^{1}$, Harry D. Bear ${ }^{1}$ and Kazuaki Takabe ${ }^{1,2,3}$

${ }^{1}$ Division of Surgical Oncology, Department of Surgery,

${ }^{2}$ Department of Biochemistry and Molecular Biology,

Virginia Commonwealth University School of Medicine and the Massey Cancer Center, Richmond, VA

${ }^{3}$ Breast Surgery, Roswell Park Cancer Institute, Buffalo NY

Short title: Breast cancer model for immunotherapy

\section{Corresponding author}

Kazuaki Takabe MD, PhD, FACS

Breast Surgery

Roswell Park Cancer Institute

Elm \& Carlton Streets

Buffalo, NY 14263

Tel: 858-945-5405, Fax: (804) 828-4808

e-mail: kazutakabe@gmail.com

\section{Authors' contributions:}

E.K., S.C.D., and K.P.T. conceptualized and performed experiments described in the article.

E.K., S.S., G.Q.P., and H.D.B. prepared the article.

K.T. provided supervision of experiments and preparation of the article. 


\section{Abstract}

Background: Interest in immunotherapy for breast cancer is rapidly emerging and applicable animal models that mimic human cancer are urgently needed for preclinical studies. This study aimed to improve a technique for orthotopic inoculation of syngeneic breast cancer cells to be used as a preclinical animal model for immunotherapy.

Materials and methods: We utilized our previously reported murine model of orthotopic cancer cell inoculation under direct vision and compared the efficiency of tumorigenesis with tumor cells suspended in either PBS or Matrigel containing varying numbers of cells. As a model for immune rejection, murine BALB/c derived 4T1-luc2 breast cancer cells were inoculated orthotopically into both BALB/c and C57BL/6 mice.

Results: Matrigel suspended cells formed larger tumors with higher efficiency than PBS suspended cells. The maximum volume of Matrigel that could be inoculated without spillage was $20 \mu \mathrm{l}$ and $30 \mu \mathrm{l}$ in the \#2 and \#4 mammary fat pads, respectively. Tumor take rates increased as the injected cell number increased. In this immune rejection model, there were no significant differences in tumor weight 
between the strains up to Day 7, after which tumor weight decreased in C57BL/6 mice. Bioluminescence in C57BL/6 mice was also significantly less than in BALB/C mice and increased up to Day 7, then swiftly decreased thereafter.

Conclusions: This improved technique of innoculating murine breast cancer cells utilizing bioluminescence technology may be useful in evaluating the efficacy of tumor regression mediated by immune responses, as shown by an allogeneic response in $\mathrm{C} 57 \mathrm{BL} / 6$ mice.

Key words: breast cancer; syngeneic model; preclinical, immunotherapy. 


\section{Introduction}

Immunotherapy, including checkpoint inhibitors, cancer vaccines, adoptive cell immunotherapy, and strategies that exploit chimeric antigen receptor engineered $\mathrm{T}$ cells, is rapidly emerging as a promising modality for different types of cancers (1). The early success of immune checkpoint inhibition, such as targeted therapy against cytotoxic T-lymphocyte-associated protein 4 (CTLA-4), along with programmed death-1 (PD-1) $(2,3)$ and the PD-1 ligand, PD-L1 (4), has drawn much attention (5).

One of the most commonly used animal models for oncologic preclinical studies is xenografts of human cancer cells or patient-derived cancer tissue into immune deficient mice $(6,7)$. However, these models are not suitable to evaluate immune responses or the effects of immunotherapy, since the host animal lacks a fully functioning immune system. Spontaneous tumorigenic models utilizing transgenic mice or carcinogens have been developed in animals with an intact immune system, but these models may require a long waiting time for the development of cancer, which limits their practicality $(8,9)$. Furthermore, these models usually require expensive equipment, such as mouse-specific imaging scanners (CT, MRI, or PET), 
to detect and measure tumors, and even with state-of-the-art diagnostic imaging equipment the evaluation of immunotherapy responses remains challenging. Tumor size may not necessary reflect the amount of cancer cells, as tumors may initially enlarge on imaging studies due to accumulated infiltrating immune cells when immunotherapy is actually effective. Recently, patient-derived "humanized" xenograft models have been developed using patient tumors implanted into immune deficient mice that are engineered to have intact human immune cells $(10-12)$. However, the cost of these animals as well as other limitations, including take rate, viral contamination and selection pressure, hinder this approach (10), and even these modern models cannot escape from the challenges of assessing tumor responses to immunotherapy (12). Thus, orthotopic inoculation of syngeneic murine tumor cells tagged with a bioluminescent reporter into immune intact mice is the most straightforward, fast, and affordable model to study the effect of immunotherapy at this point.

We have previously reported the establishment of a murine syngeneic breast cancer model utilizing cell inoculation into chest mammary fat pads under direct vision, which can mimic human cancer progression (13-15). In this study, we report 
the establishment of an improved orthotopic inoculation technique of murine breast cancer cells using luciferase-tagged 4T1-luc2 murine cancer cells suspended in Matrigel, and demonstrate that this model is useful to assess immune-mediated regression of breast tumors.

\section{Materials and Methods}

\subsection{Cell culture}

4T1-luc2 cells, a mouse mammary adenocarcinoma cell line derived from BALB/c mice that has been engineered to express luciferase was purchased from Caliper Life Sciences/PerkinElmer (Hopkinton, MA). 4T1-luc2 cells were cultured in RPMI Medium 1640 with 10\% fetal bovine serum (FBS). E0771 cells, a C57BL/6 mouse mammary fat pad-derived adenocarcinoma cell line were cultured in DMEM with $10 \%$ FBS.

\subsection{Animal models}

Approval from the Virginia Commonwealth University Institutional Animal Care and Use Committee was obtained for all experiments. Female BALB/c and C57BL/6 
mice were obtained from Jackson Laboratory. All cell inoculations into \#2 (chest) and \#4 (abdomen) mammary fat pads under direct vision were carried out as previously described (13). All procedures were performed utilizing sterile technique under isoflurane anesthesia and the animals were prepped and draped in a sterile manner. In brief, a $10 \mathrm{~mm}$ incision was made medial to the nipple and a cotton swab was used to expose the mammary gland. A syringe with a $26 \mathrm{G}$ needle was used to inject the cell suspension directly into the mammary gland under direct vision, and the wound was closed with a suture. To maximize the take rate, cell inoculations were conducted within 1 hour from preparation of cell suspensions. Tumor growth was monitored by caliper measurement and animals were weighed every other day.

\subsubsection{Preparation of Matrigel and PBS cell suspensions}

$1 \times 10^{4} 4 \mathrm{~T} 1$-luc2 cells were suspended in $20 \mu \mathrm{l}$ of either PBS or Matrigel. Cells were inoculated into \#2 and \#4 mammary fat pads of BALB/c mice $(n=8) .14$ days after inoculation, tumors were assessed by palpation, then harvested and weighed.

\subsubsection{Determination of the optimum amount of Matrigel for injection into}




\section{mammary fat pads}

To determine the amount of Matrigel a mammary fat pad can hold, $10 \mu \mathrm{l}$ of Matrigel stained with $10 \%$ trypan blue were injected incrementally into the \#2 and \#4 mammary fat pads ( $n=12$, each group). Spillage of Matrigel out of the fat pad was assessed visually after each injection.

\subsubsection{Tumorigenesis with different numbers of cells inoculated}

4T1-luc2 cells $\left(5 \times 10^{2}, 1 \times 10^{3}, 5 \times 10^{3}, 1 \times 10^{4}\right)$ or E0771 cells $\left(5 \times 10^{4}, 1 \times 10^{5}, 5 \times 10^{5}\right.$, $1 \times 10^{6}$ ) were suspended in $20 \mu \mathrm{l}$ of Matrigel, then inoculated into the \#2 and \#4 mammary fat pads of C57BL/6 or BALB/c mice, respectively ( $n=8$, each group). Formation of tumors was determined by palpation. Larger numbers of E0771 cells were inoculated because of the slower growth rate of this tumor in the appropriate syngeneic mouse strain.

\subsubsection{T1-luc2 tumors in C57BL/6 mice (immune rejection model)}

$1 \times 10^{4}$ of $4 \mathrm{~T} 1-\mathrm{luc} 2$ cells, derived from BALB/c mice, suspended in Matrigel were implanted into the right \#2 fat pad of $\mathrm{C} 57 \mathrm{BL} / 6$ mice or BALB/c mice as a control. 
Tumor growth was monitored every other day by bioluminescence (IVIS) imaging ( $\mathrm{n}$

= 4). For tumor weight analysis, cells were inoculated into bilateral \#2 and \#4 fat pad and tumors were harvested and weighed on Days 3,7 , and $9(n=8$, each). Pathological analyses were performed after formalin fixation of the tumors. Tumor sections were prepared and stained with hematoxylin and eosin (H\&E) by Virginia Commonwealth University Health System Anatomic Pathology Research Services.

\subsection{Bioluminescent quantification of tumor burden}

Xenogen IVIS 200 and Living Image software (Caliper Life Sciences/PerkinElmer) were used to quantify the photon/sec emitted by $4 \mathrm{T1}-\mathrm{luc2}$ cells. D-Luciferin (150 mg/kg; PerkinElmer) was injected intraperitoneally into mice previously implanted with 4T1-luc2 cells. Bioluminescence was measured and quantified at 5 minute intervals up to photo-count peak. Bioluminescence was then determined by the peak number of photons/sec calculated over this time frame (15).

\subsection{Statistical analyses}

Statistical analyses were performed by the chi-square test or the Fisher exact 
test with a single degree of freedom, and the Student's t test was used to analyze the differences between two groups. $P$ values less than 0.05 were considered to have statistical significance. All statistical analyses were performed using SPSS version 23.0 (SPSS, Chicago, IL).

\section{Results}

\subsection{Comparison of tumorigenesis after inoculation of cell suspensions in}

\section{Matrigel or PBS}

The locations of mouse mammary fat pads are illustrated in Fig. 1A after injection of $10 \%$ trypan blue dye through the skin close to the nipple. The image shows the relative ease of access to \#2 and \#4 mammary fat pads for inoculation under direct vision from exposure through a midline incision. Notable in Fig. IA, \#1 pads are not visible despite wide exposure, \#3 pads are too small to access, and \#5 pads are too deep at the base of the legs for accurate inoculation. Therefore, \#2 and \#4 mammary fat pads were used for orthotopic inoculation of breast cancer cells in subsequent experiments.

In our previous study, we found that inoculation of cell suspensions in PBS 
resulted in spillage out of the mammary fat pads, with scatter of cells outside the pads (13). Therefore, the volume injected was minimized, which limited the number of cells that could be inoculated. We now have compared the efficiency of generating 4T1-luc2 tumors from cells suspended in Matrigel with that of PBS suspended cells in our orthotopic inoculation under direct vision model. Only 3 out of 8 mice $(37.5 \%)$ developed palpable tumors from cells injected in PBS, whereas all mice $(8$ of $8,100 \%)$ developed tumors after inoculation of tumor cells suspended in Matrigel by 2 weeks (Fig. 1B). Tumors generated from Matrigel suspended cells weighed significantly more than from cells suspended in PBS $(p<0.001)$ (Fig. 1C). In agreement with our previous observations (13), inoculation of PBS suspended cells resulted in cancer cells forming tumors not only in the mammary fat pad, but also in subcutaneous tissue outside of the mammary fat pad (Fig. 1D left panel) In contrast, Matrigel suspended cells formed single tumors confined to the mammary fat pad (Fig. 1D right panel). These results demonstrate that cells suspended in Matrigel are more efficient at generating a discrete primary breast tumor in our orthotopic model. 


\subsection{Determination of the amount of Matrigel a mammary fat pad can hold}

We then investigated the amount of Matrigel that the \#2 or \#4 mammary fat pads can hold without spillage. The amount of injectate (trypan blue stained Matrigel) was incrementally increased by $10 \mu$ until spillage occurred. As shown in Fig. 2A and 2B, spillage was observed when more than $20 \mu \mathrm{l}$ or $30 \mu \mathrm{l}$ of Matrigel were injected into the \#2 or \#4 mammary fat pads, respectively. Therefore, $20 \mu l$ and $30 \mu l$ of Matrigel were identified to be the most suitable amounts to inject into \#2 or \#4 fat pads, respectively.

\subsection{Dependence of tumor formation on number of cells inoculated}

To examine the relationship between inoculated cell number and generation of tumors, increasing numbers of cells were inoculated into the \#2 or \#4 mammary fat pads and tumorigenesis monitored. We utilized the two most commonly used syngeneic breast cancer models, 4T1-luc2 cells into BALB/c mice and E0771 cells into $\mathrm{C} 57 \mathrm{BL} / 6$ mice. We found that tumorigenesis increased as the inoculated cell number increased for both cell lines, where all animals developed a tumor with 5x10 4 T1-luc2 cells, and $1 \times 10^{6}$ E0771 cells (Fig. 3A, 3B). 


\subsection{Tumor growth in different background mice}

To investigate whether our model is appropriate to assess immune-mediated regression of breast tumors, we examined the growth of 4T1-luc2 cells, inoculated in Matrigel, in an environment where the cells will be rejected by an allogeneic immune response. 4T1-luc2 cells were orthotopically inoculated into either syngeneic $(\mathrm{BALB} / \mathrm{c})$ or allogeneic $(\mathrm{C} 57 \mathrm{BL} / 6)$ mice. Thus, the tumors growing in C57BL/6 mice would be expected to be destroyed by the native immune system. The number of viable cancer cells and tumor burden were monitored in vivo by bioluminescence (Fig. 4A). Two-fold increases in tumor growth measured by bioluminescence imaging were nearly identical in both backgrounds 24 hour after inoculation. By 7 days after inoculation, 4T1-luc2 cells in C57BL/6 mice grew approximately 160-fold their inoculated amount, whereas the tumors in BALB/c mice grew 1200-fold their inoculated amount (Fig. 4B). The BALB/c tumors continued to grow rapidly to reach an almost 10,000-fold increase in bioluminescence signal, while the C57BL/6 mice tumors swiftly decreased the signal from Day 7 and the viable cancer cells were eliminated by Day 14 . We further 
analyzed the time after injection of luciferin required to reach a peak photo-count, which is presumably reflective of perfusion of the tumor. Time to reach a peak photo-count declined by Day 7 in both BALB/c and C57BL/6 mice, after which it continued to decline in C57BL/6 tumor by Day 13, whereas it increased after Day 10 in BALB/c mice, most likely reflecting the growth of the tumor.

There were no significant differences in tumor weights at Days 3 and 7 between tumors in BALB/c and C57BL/6, but a significant difference was observed by Day 9 $(p=0.0132)($ Fig. $5 \mathrm{~A})$. Histological analysis showed that cancer cells were surrounded by inflammatory cells on Day 9 in the C57BL/6 mice and were destroyed by Day 15 in the C57BL/6 mice, whereas cancer cells without inflammatory infiltrate filled the tumor in BALB/c mice (Fig. 5B).

\section{Discussion}

For the last 8 years, our laboratory has been studying murine cancer models that mimic human cancer progression to be used for preclinical trials (6). Our conclusion to date is that no murine model perfectly mimics human cancer progression; thus, multiple models should be used for preclinical studies to 
supplement each other's weaknesses $(6,7)$. Given the essential role of immune cells and inflammation in cancer progression, the preclinical results obtained using immune deficient mice can be misleading and may be contributing to the low success rate of anti-cancer drug development (6). Our prior work has shown that inoculation of syngeneic mouse cancer cells orthotopically into the mammary fat pads of immune intact mice under direct vision provides us with the most stable results that mimic human cancer progression $(13,16)$. This was also shown in a murine model of colon cancer, where cell suspension in Matrigel was found to be efficacious (17). Together with previous reports that cell suspension in Matrigel facilitates the establishment of tumors in xenograft models (18), our preliminary work led us to the current study to improve our breast cancer model using Matrigel. Compared with cells suspended in PBS, cells suspended in Matrigel developed tumors more efficiently and were more representative of primary breast tumors. We also identified the appropriate amount of Matrigel to be injected without spillage into the \#2 and \#4 mammary fat pads, and found that the take rate correlated with the cell number inoculated. To our knowledge, this is the first to report this type of improved model utilizing Matrigel for luciferase-tagged syngeneic breast cancer cell 
inoculation in the mammary fat pad under direct vision.

In order to test whether our improved model is suitable for preclinical evaluation of immunotherapy, we decided to investigate 4T1-luc2 cell growth in a model invoking immune rejection, namely implantation of murine cancer cells into immune intact allogeneic mice. BALB/c derived 4T1-luc2 cells continued to proliferate in C57BL/6 mice for one week prior to being eliminated, most likely reflecting the time to develop a primary immune response. Of note, the peak photon counts quantified by bioluminescence in immune compatible BALB/c mice were approximately 12 -fold and 240-fold higher compared from C57BL/6 mice on Day 3 and Day 7, respectively. Strikingly, there were no significant differences in tumor size between BALB/c and C57BL/6 mice on Day 3 and Day 7. Histological analyses revealed that there were numerous infiltrating lymphocytes in the $4 \mathrm{~T} 1$ tumors implanted in C57BL/6 mice, whereas predominantly cancer cells were seen in BALB/c mice on Day 9, suggesting that the tumor size in the C57BL/6 mice was maintained in part by tumor-infiltrating immune cells in the process of eliminating the cancer. Indeed, on Day 15 , no viable cancer cells were detected in the mammary pad of C57BL/6 mice. We also measured the time to reach the peak photon counts quantified by 
bioluminescence after intraperitoneal injection of D-luciferin, which is reflective of diffusion of the substrate into cancer cells. In agreement with a previous report (19), the time to peak is prolonged after Day 7 in BALB/C tumors following the enlargement of the tumor, while the time to peak continued to shorten in C57BL/6 tumor due to the tumor shrinkage.

One of the challenges of immunotherapy research is the method to evaluate the response to treatment. Commonly the effect of systemic chemotherapy is evaluated by RECIST (Response Evaluation Criteria in Solid Tumors) criteria, which relies on tumor size reduction (20). In contrast with chemotherapy's immediate and direct cytotoxic and tumor shrinkage effect, immunotherapy stimulates the patient's immune system to mount an antitumor response, which includes infiltration of immune-related cells into the tumor. This often results in a paradoxical transient increase in tumor size, which can mislead the physician to perceive this as treatment failure and result in premature termination of an effective immunotherapy. This is also the case in preclinical animal studies where viable cancer cells and not the size of the tumors need to be monitored. This paradox was observed in the current study as well, when the cancer cell number monitored by bioluminescence 
was significantly lower in the C57BL/6 immune rejection model when compared to BALB/c model, while the measured tumor sizes were not different. This further highlights the benefits of our luciferase-tagged syngeneic cell inoculation model, which could be used to assess immunotherapy for breast cancer, such as adoptive immunotherapy, cancer vaccines or immune checkpoint inhibitors.

\section{Conclusions}

In conclusion, we have established an improved murine orthotopic model using Matrigel, in combination with bioluminescence technology which may be useful for preclinical studies of immunotherapy.

\section{Acknowledgement}

This work was supported by NIH grant R01CA160688 and Susan G. Komen Foundation Investigator Initiated Research grant (IIR12222224) to K.T.

\section{Disclosure:}

There are no potential conflicts of interest to disclose. 


\section{Figure Legend}

Figure 1. Location of mouse mammary fat pads and the difference in tumorigenesis of cancer cells suspended in Matrigel or PBS

(A) The locations of mouse mammary fat pads (\#2-5) were identified after midline incision. This representative image depicts the ease to access \#2 and \#4 mammary fat pads for inoculation under direct vision. Note, \#1 pads cannot be seen and \#3 pads are too small, and $\# 5$ pads are too deep at the base of the legs for accurate inoculation. (B) Tumorigenesis 2 weeks after inoculation of $1 \times 10^{4} 4$ T1-luc2 cells. (C) Tumor weights were significantly greater in the tumors from Matrigel suspended cells, than from the PBS suspended cells. $(n=8)(D)$ Histologic examination showed that inoculation of PBS suspended cells resulted in scattering of cancer cells in the mammary fat pad and cancer cells were also seen in secondary tumors outside the mammary fat pad, whereas Matrigel suspended cells formed single tumors confined to in the mammary fat pad.

Figure 2. The amount of Matrigel a mammary fat pad can hold without spillage. 
Trypan blue stained Matrigel was injected into mammary fat pads in $10 \mu \mathrm{l}$ increments and spillage was detected visually. (A) Representative images of Trypan Blue stained Matrigel inoculation. Spillage out of the mammary fat pads is observed

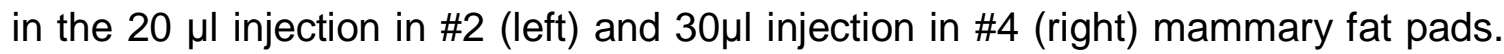
(B) The amount of Matrigel that began to spill out of the pads when inoculated to either \#2 or \#4 mammary fat pads.

Figure 3. Number of cells in Matrigel inoculates required to generate palpable tumors in two syngeneic breast cancer models.

Shown on the $\mathrm{X}$ axes are the numbers of 4T1-luc2 cells inoculated into mammary fat pads of BALB/c mice $(n=8)(A)$ and E0771 cells inoculated into C57BL/6 mice $(n=8)(B)$. The proportion of mice with palpable tumors (Tumorigenesis) was assessed at 1 and 2 weeks after inoculation for 4T1-luc2 and 2 and 3 weeks for E0771.

Figure 4. 4T1-luc2 breast cancer growth after orthotopic inoculation into the mammary pads of BALB/c or C57BL/6 mice. 
$\mathrm{BALB} / \mathrm{c}$ or $\mathrm{C} 57 \mathrm{BL} / 6$ mice were orthotopicaly inoculated with $1 \times 10^{4} 4 \mathrm{~T} 1$-luc2 cells under direct vision, and tumor growth was evaluated by bioluminescence every other day. (A) Representative bioluminescence images of 4T1-luc2 tumors after inoculation in $\mathrm{BALB} / \mathrm{c}$ or $\mathrm{C} 57 \mathrm{BL} / 6$ mice that showed tumor growth during observation period. (B) Tumor burden determined by photon counts was measured every other day $(n=4$, mean \pm SEM). (C) The time required to reach a peak photocount continued to decline in C57BL/6 mice, whereas it increased by Day 10 in BALB/c mice.

Figure 5. Tumor weights and histological analyses of 4T1-luc2 tumors in BALB/C and C57BL/6 mice.

(A) Tumors were harvested and weighed on Day 3, 7, and 9 ( $\mathrm{n}=8$, each). There were no significant differences in tumor weights on days 3 and 7 between tumors in $\mathrm{BALB} / \mathrm{C}$ and C57BL/6. In contrast, a significant difference was observed on Day 9. (B) Hematoxylin and eosin staining of 4T1-luc2 tumors in BALB/C and C57BL/6 mice. Cancer cells were surrounded by inflammatory cells on Day 9, and cancer cells were not seen on Day15 in the tumor site of C57BL/6 mice. 


\section{References}

1. Mick R, Chen TT Statistical Challenges in the Design of Late-Stage Cancer Immunotherapy Studies. Cancer Immunol Res 2015:3:1292-1298.

2. Topalian SL, Hodi FS, Brahmer JR, Gettinger SN, Smith DC, et al. Safety, activity, and immune correlates of anti-PD-1 antibody in cancer. $\mathrm{N}$ Engl $\mathrm{J}$ Med 2012:366:2443-2454.

3. Hamid O, Robert C, Daud A, Hodi FS, Hwu WJ, et al. Safety and tumor responses with lambrolizumab (anti-PD-1) in melanoma. $\mathrm{N}$ Engl $\mathrm{J}$ Med 2013:369:134-144.

4. Brahmer JR, Tykodi SS, Chow LQ, Hwu WJ, Topalian SL, et al. Safety and activity of anti-PD-L1 antibody in patients with advanced cancer. N Engl J Med 2012:366:2455-2465.

5. Couzin-Frankel $\mathrm{J}$ Breakthrough of the year 2013. Cancer immunotherapy. Science. United States: 2013:1432-1433.

6. Rashid OM, Takabe K Animal models for exploring the pharmacokinetics of breast cancer therapies. Expert Opin Drug Metab Toxicol 2015:11:221-230.

7. Rashid OM, Takabe $\mathrm{K}$ Does removal of the primary tumor in metastatic 
breast cancer improve survival? J Womens Health (Larchmt) 2014:23:184-188.

8. Hait NC, Avni D, Yamada A, Nagahashi M, Aoyagi T, et al. The phosphorylated prodrug FTY720 is a histone deacetylase inhibitor that reactivates ERalpha expression and enhances hormonal therapy for breast cancer. Oncogenesis 2015:4:e156.

9. Liang J, Nagahashi M, Kim EY, Harikumar KB, Yamada A, et al. Sphingosine-1-phosphate links persistent STAT3 activation, chronic intestinal inflammation, and development of colitis-associated cancer. Cancer Cell 2013:23:107-120.

10. Whittle JR, Lewis MT, Lindeman GJ, Visvader JE Patient-derived xenograft models of breast cancer and their predictive power. Breast Cancer Res 2015:17:17. 11. Lawson DA, Bhakta NR, Kessenbrock K, Prummel KD, Yu Y, et al. Single-cell analysis reveals a stem-cell program in human metastatic breast cancer cells. Nature 2015:526:131-135.

12. Aparicio S, Hidalgo M, Kung AL Examining the utility of patient-derived xenograft mouse models. Nat Rev Cancer 2015:15:311-316.

13. Rashid OM, Nagahashi M, Ramachandran S, Dumur C, Schaum J, et al. An 
improved syngeneic orthotopic murine model of human breast cancer progression. Breast Cancer Res Treat 2014:147:501-512.

14. Rashid OM, Nagahashi M, Ramachandran S, Graham L, Yamada A, et al. Resection of the primary tumor improves survival in metastatic breast cancer by reducing overall tumor burden. Surgery 2013:153:771-778.

15. Nagahashi M, Ramachandran S, Kim EY, Allegood JC, Rashid OM, et al. Sphingosine-1-phosphate produced by sphingosine kinase 1 promotes breast cancer progression by stimulating angiogenesis and lymphangiogenesis. Cancer Res 2012:72:726-735.

16. Rashid OM, Nagahashi M, Ramachandran S, Dumur Cl, Schaum JC, et al. Is tail vein injection a relevant breast cancer lung metastasis model? J Thorac Dis 2013:5:385-392.

17. Terracina KP, Aoyagi T, Huang WC, Nagahashi M, Yamada A, et al. Development of a metastatic murine colon cancer model. J Surg Res 2015:199:106-114.

18. Mullen $\mathrm{P}$ The use of Matrigel to facilitate the establishment of human cancer cell lines as xenografts. Methods Mol Med 2004:88:287-292. 
19. Cui $\mathrm{K}, \mathrm{Xu} \mathrm{X}, \mathrm{Zhao} \mathrm{H}$, Wong ST A quantitative study of factors affecting in vivo bioluminescence imaging. Luminescence 2008:23:292-295.

20. Eisenhauer EA, Therasse P, Bogaerts J, Schwartz LH, Sargent D, et al. New response evaluation criteria in solid tumours: revised RECIST guideline (version 1.1). Eur J Cancer 2009:45:228-247. 
Figure 1

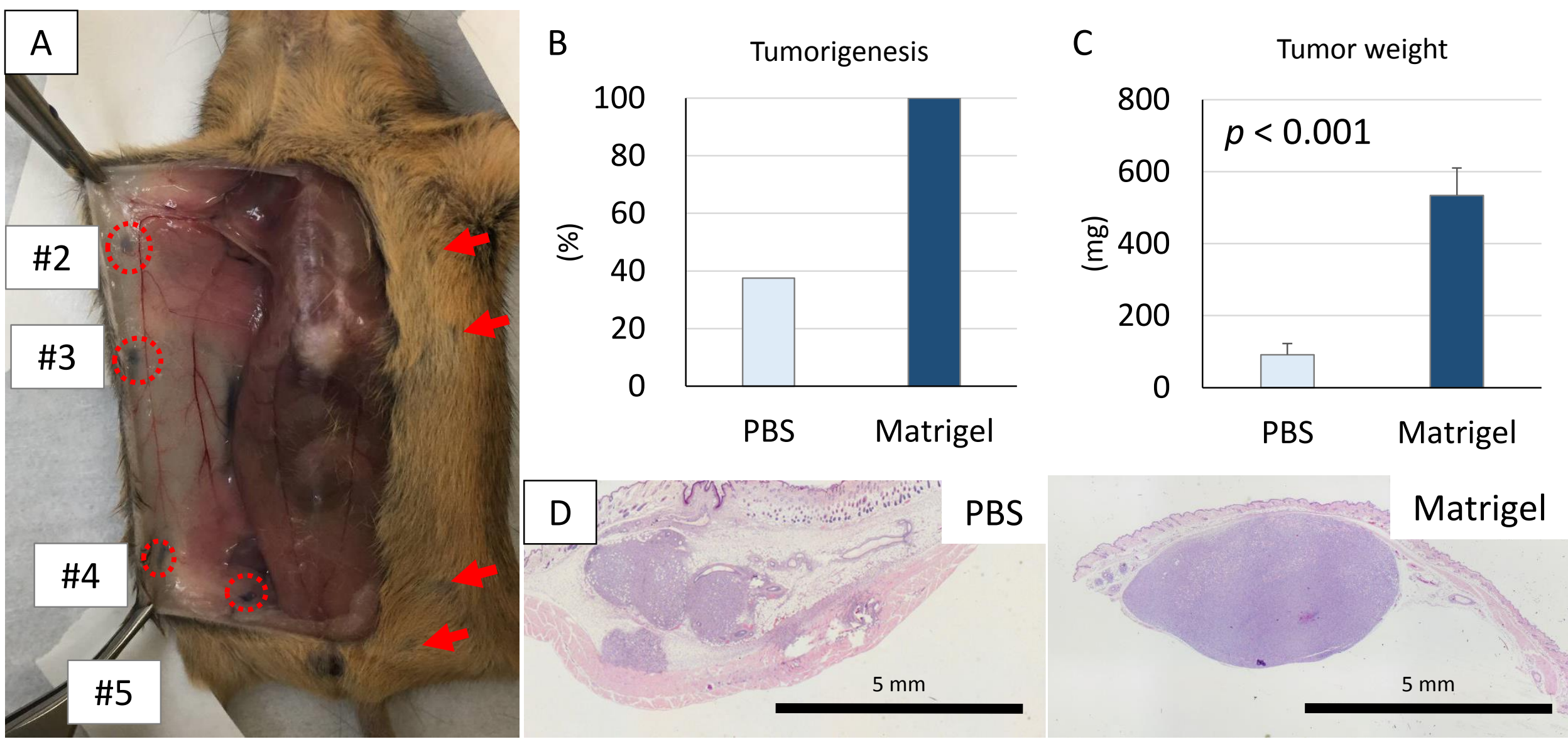


Figure 2

A

\#2

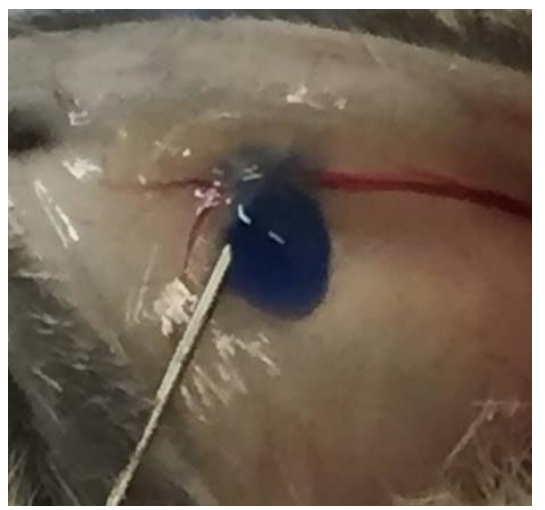

Injected $40 \mu$ l

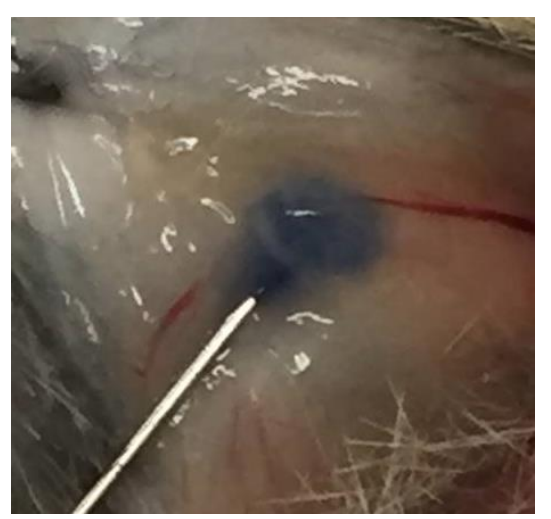

Injected $20 \mu \mathrm{l}$
\#4

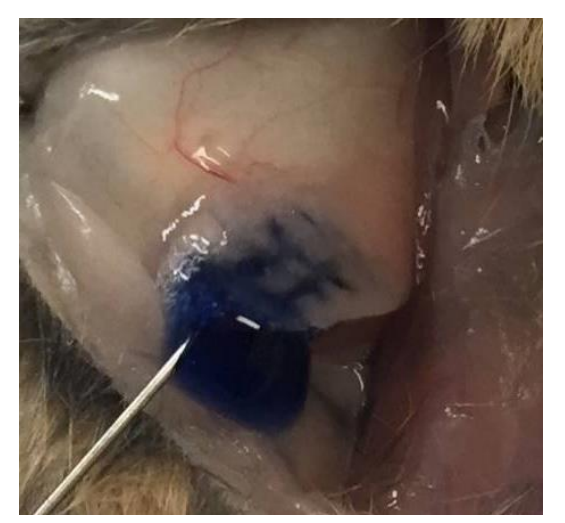

Injected $60 \mu \mathrm{l}$

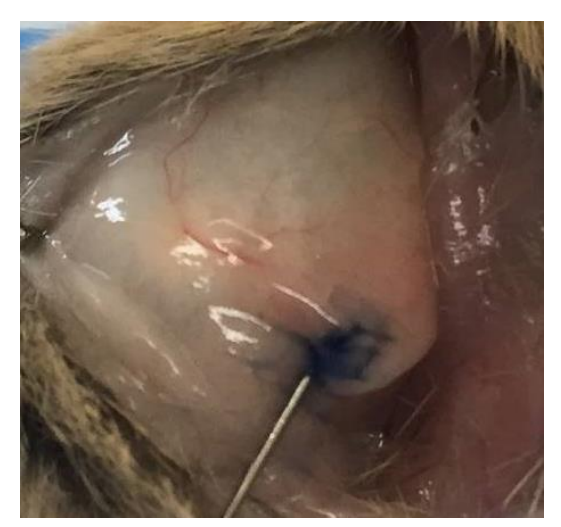

Injected $30 \mu \mathrm{l}$
B The amount of injected Matrigel starting spillage

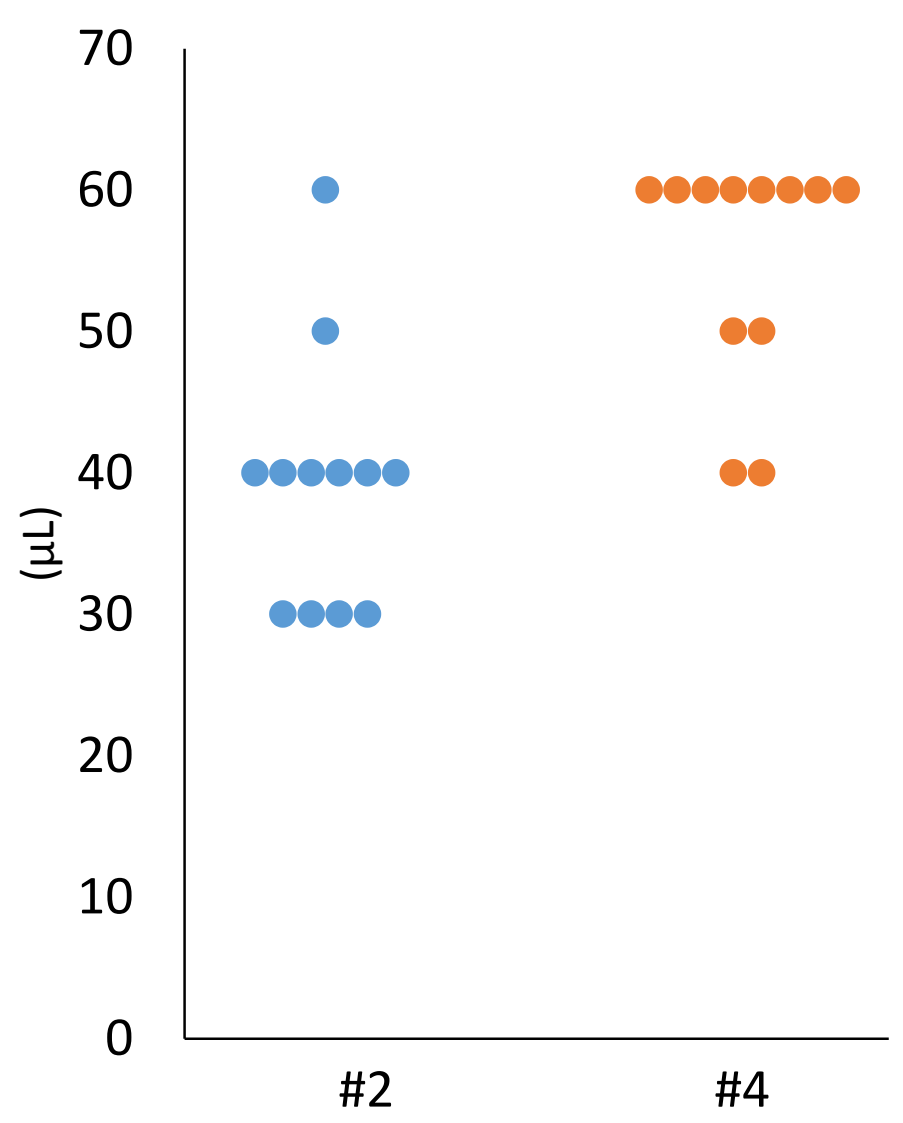


Figure 3
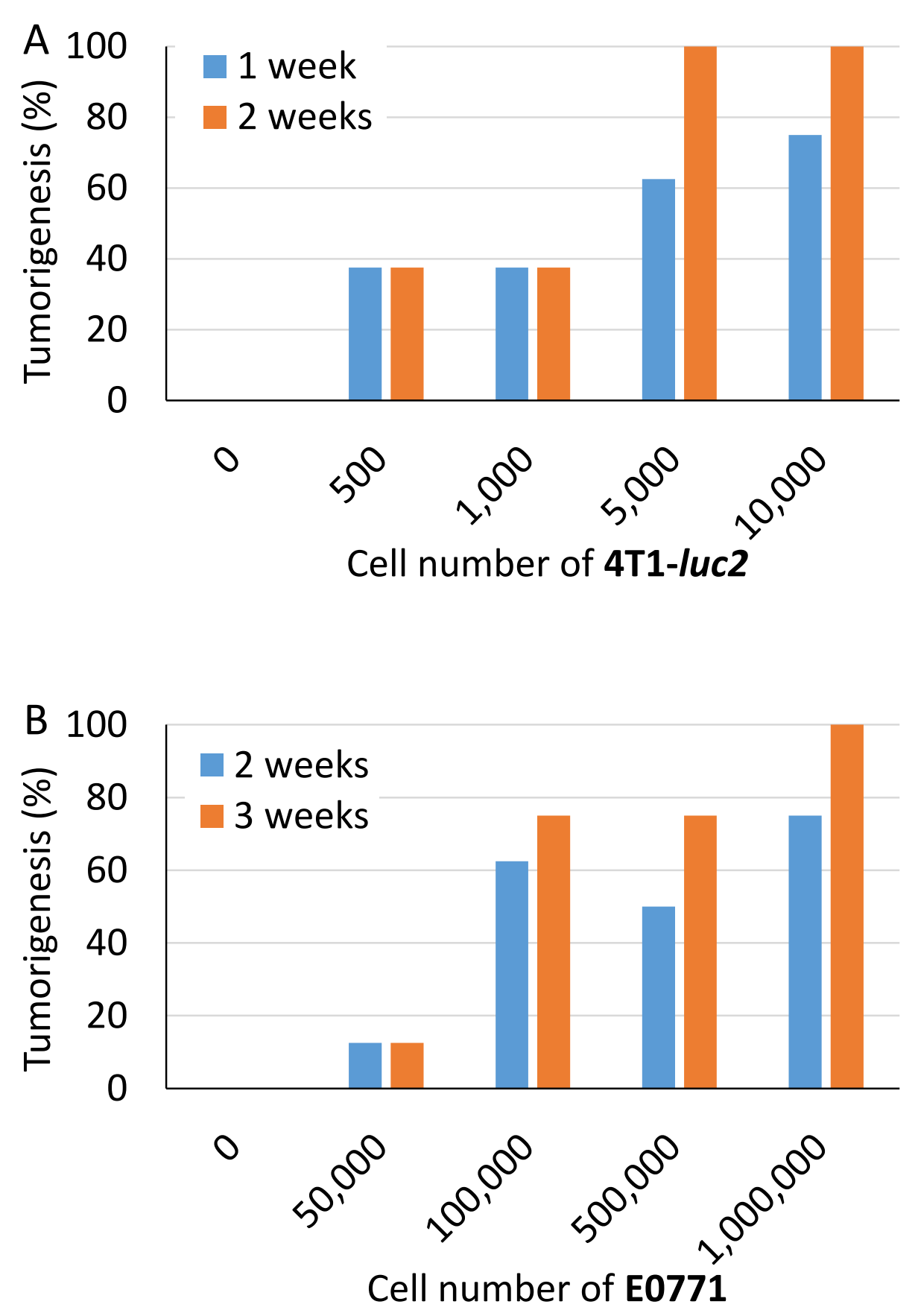
Figure 4
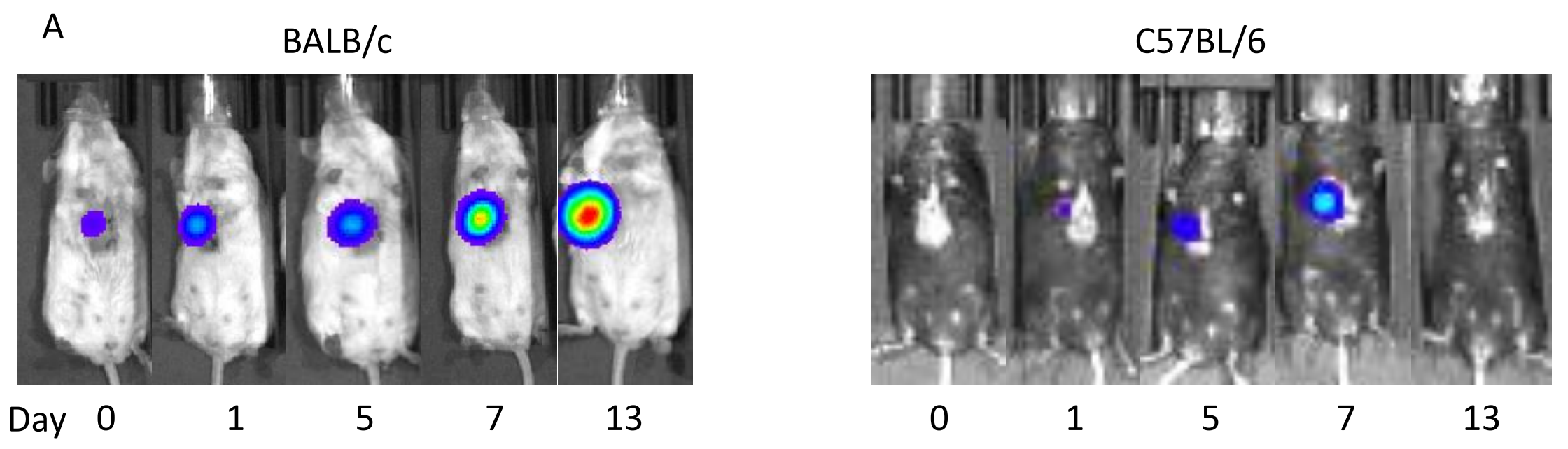


\section{Figure 4}

B

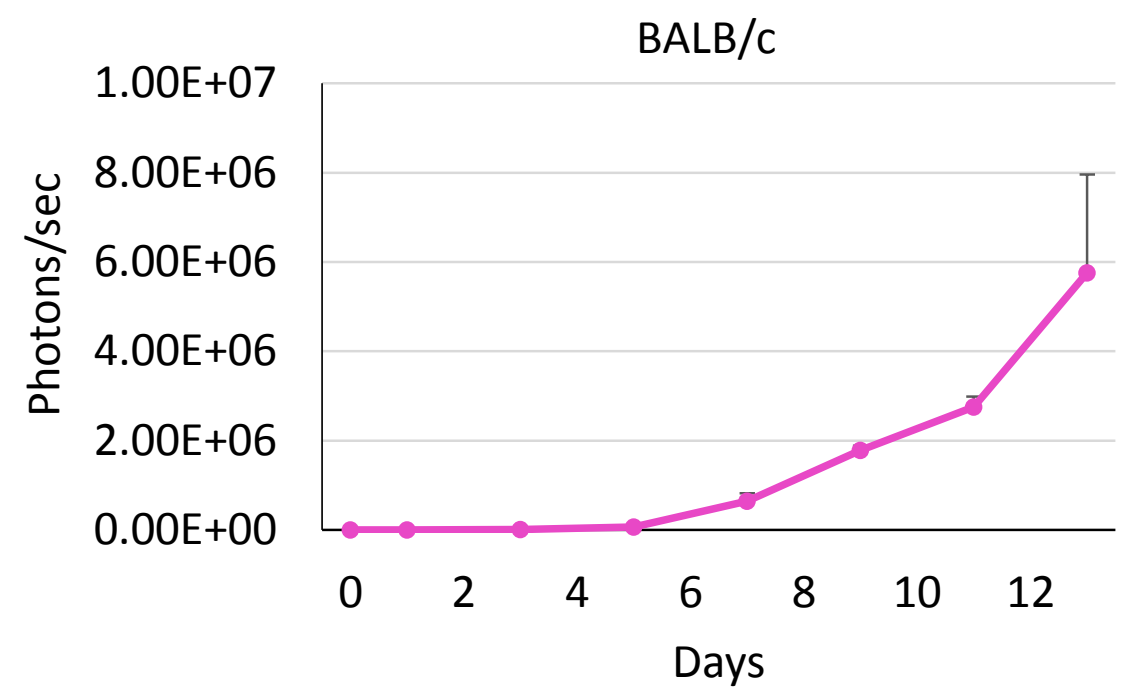

C

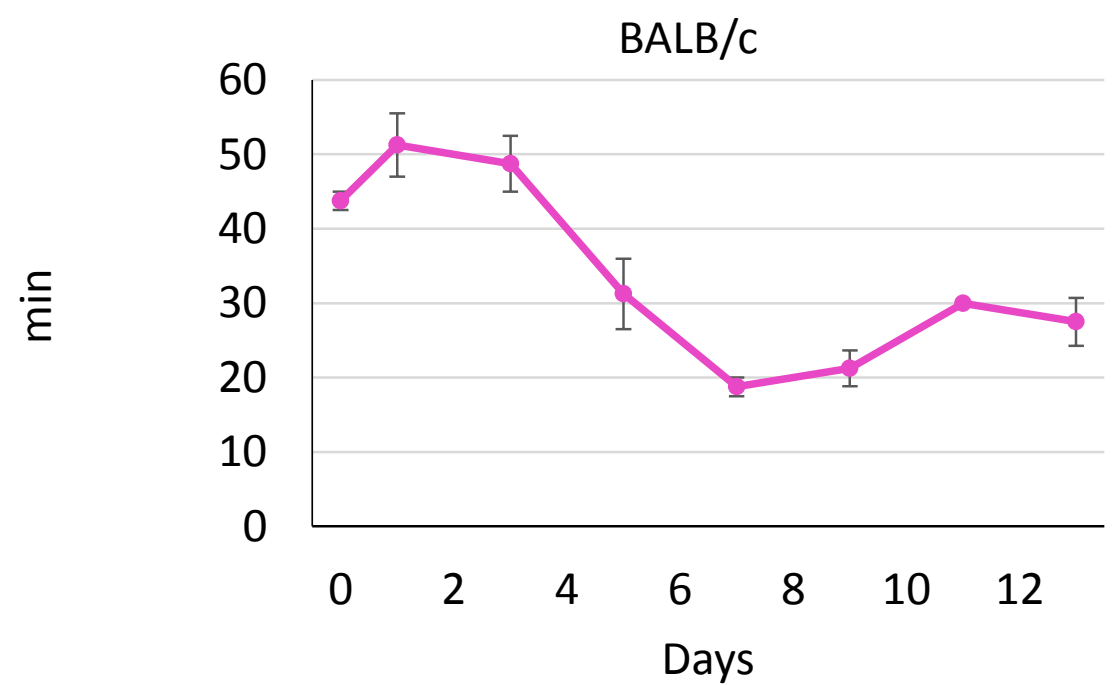

Peak photocount

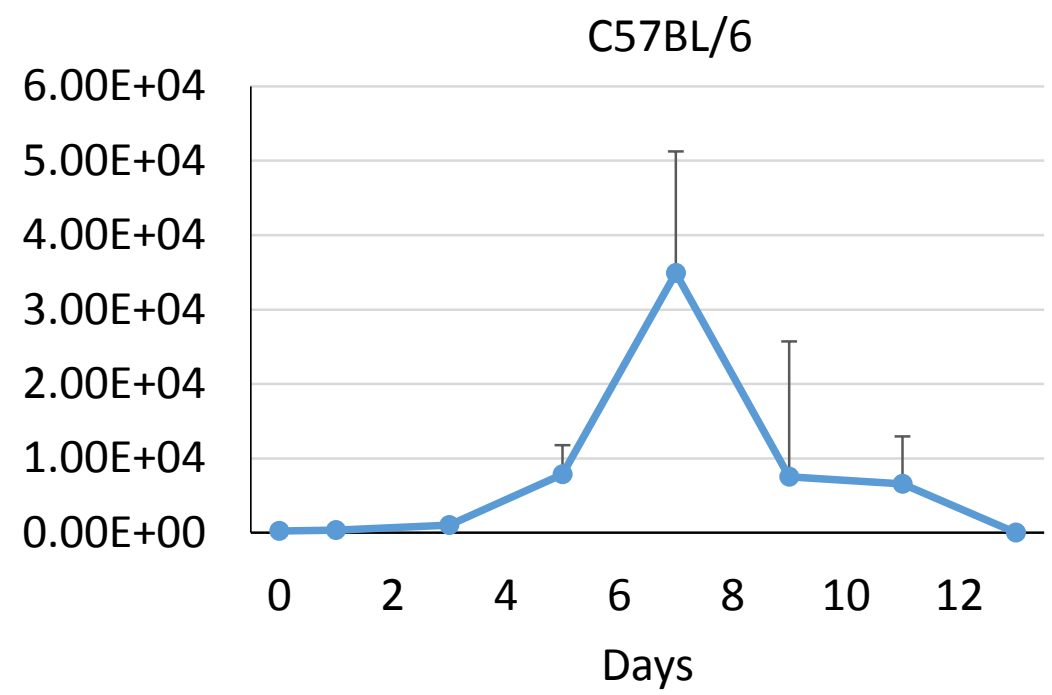

Peak time

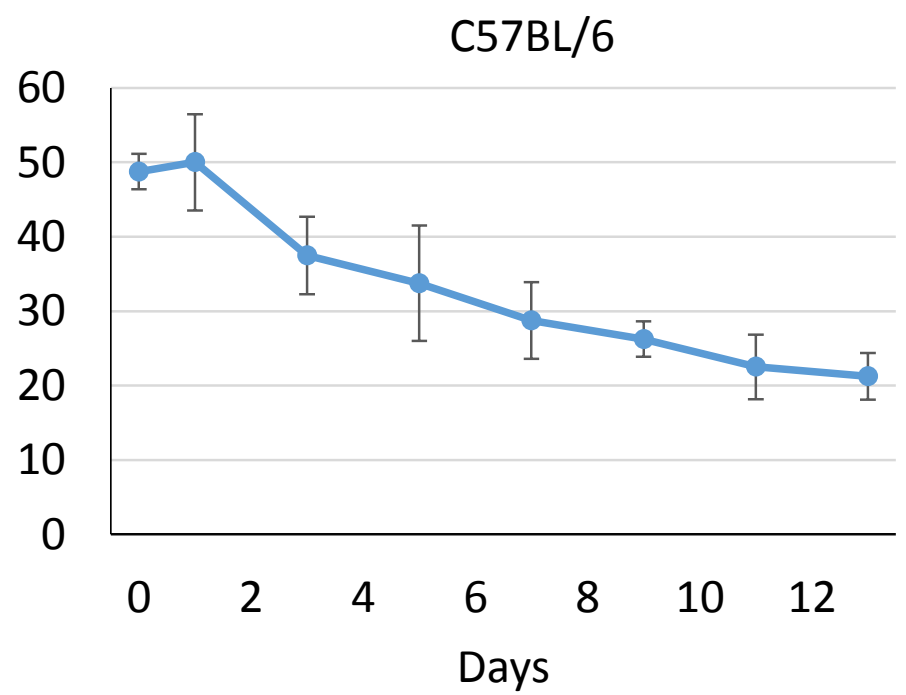


Figure 5

A

Tumor weight
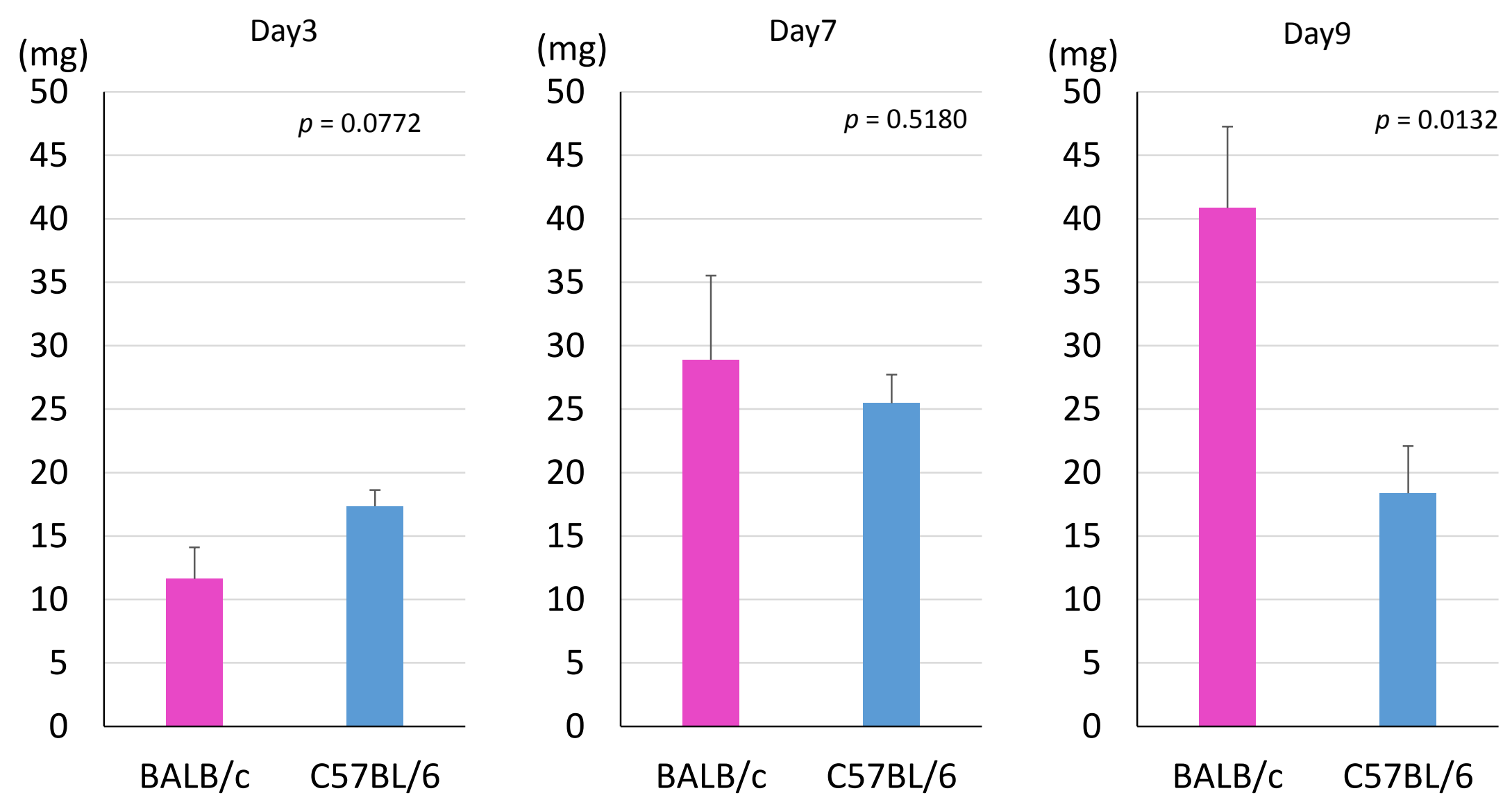


\section{Figure 5}

B
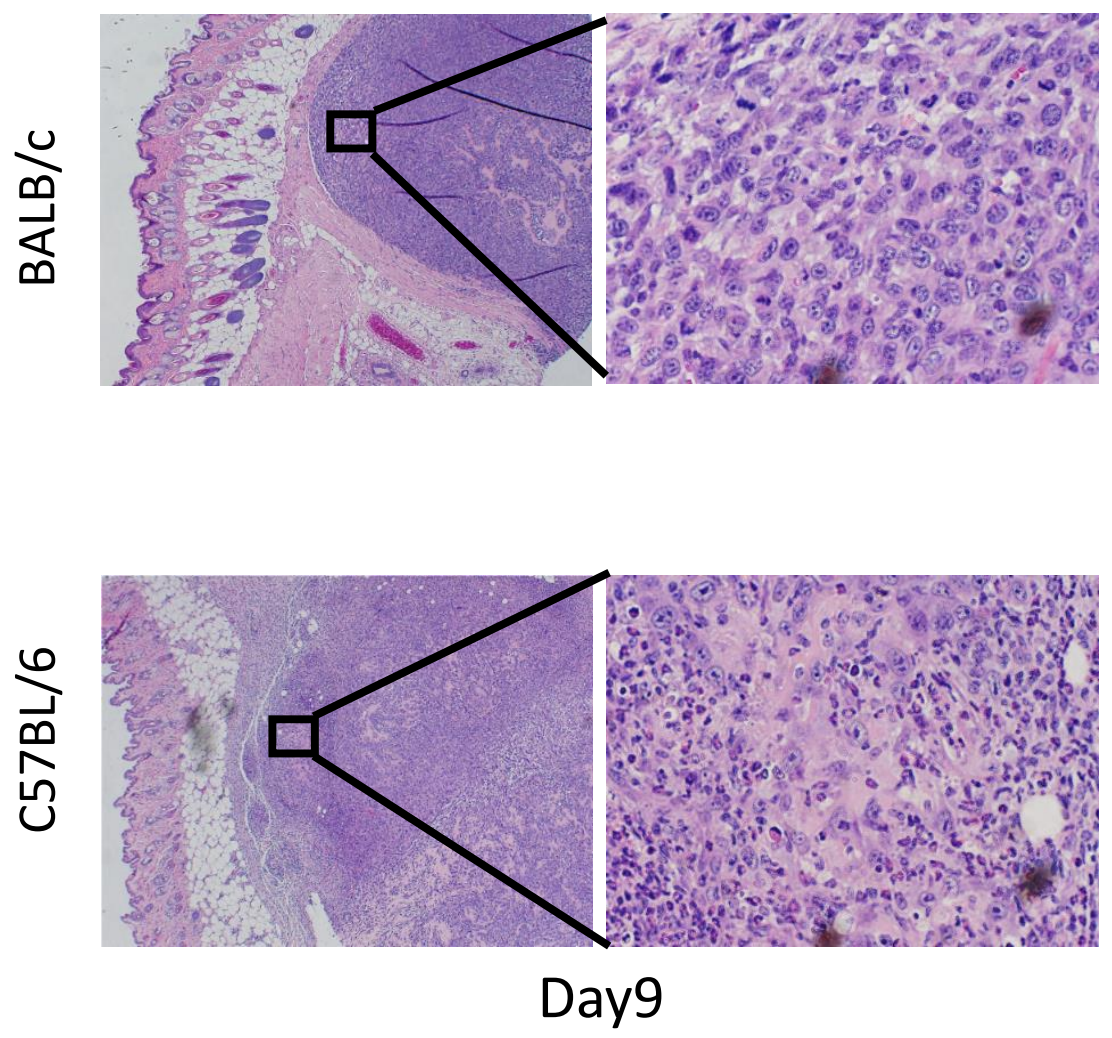
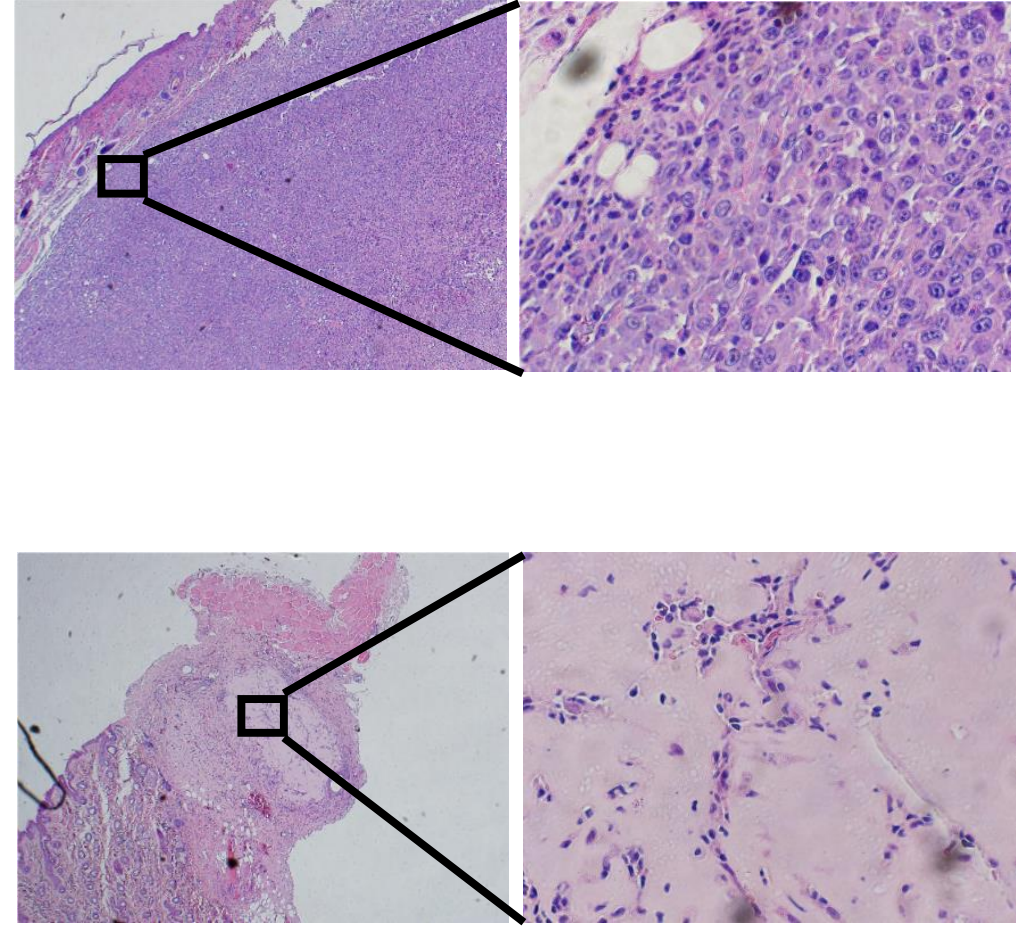

Day15 